\title{
SOBRE LA LITERATURA DE VIAJES Y LOS VIAJEROS. OLIVIA M. STONE: APROXIMACIÓN A UNA BIOGRAFÍA DESCONOCIDA
}

\author{
Daniel García Pulido \\ Biblioteca General y de Humanidades, Universidad de La Laguna
}

\section{RESUMEN}

La figura de Olivia M. Stone, con su obra Tenerife and Its Six Satellites, constituye uno de los referentes dentro de la literatura histórica de viajes relativa a las Islas Canarias. Su libro ocupa meritoriamente una posición de privilegio en dicha bibliografía foránea al tratarse de una de las primeras obras en reflejar la descripción y visita puntual a todas y cada una de las siete islas, así como por la reseñable variedad de registros recopilados por la incansable viajera, con referencias que van desde el ámbito etnográfico, gastronómico o climatológico a detallar pautas históricas, sociodemográficas o sencillamente anecdóticas. Este artículo pretende adentrarse en las claves biográficas de Olivia Mary Hartrick Stone que aún permanecían inéditas, para conocer más y mejor a la autora de tan magna obra.

Palabras clave: literatura de viajes, biografía, siglo xix, Islas Canarias, Olivia M. Stone, Tenerife and Its Six Satellites.

ON TRAVEL LITERATURE AND TRAVELLERS.

OLIVIA M. STONE: AN APPROACH TO AN UNKNOWN BIOGRAPHY

\section{Abstract}

Tenerife and its Six Satellites by Olivia M. Stone is one of the main works of reference in the field of historical travel literature of the Canary Islands. The book rightly occupies a privileged place in the foreign bibliography of the Canary Islands, since it is one of the first in which can be found not only the description of a visit to each of the seven islands, but also a wide variety of observations made by this tireless traveller, whose notes range from ethnography, gastronomy and climate, to history, demographics and amusing anecdotes. The aim of this article is to bring some unknown details of biographical information about Olivia Mary Hartrick Stone to the researchers and readers in order to know more and to understand better the author of such a relevant work.

KeYwords: Travel literature, biography, $19^{\text {th }}$ century, Canary Islands, Olivia M. Stone, Tenerife and Its Six Satellites. 
The scene is one of exquisite beauty. Basaltic precipices are upon two sides, a silvery strand, backed by a cliff of sand, forming the third. The sea is deep blue, and upon its rippling surface, in the shelter of the bay, float white-plumaged sea-fowl and a boat. The sky above is blue; the sun is shining. Verily we have reached the Isles of the Blest.

O.M.S.

Para quien se adentra en el estudio y la lectura de las obras clásicas de la literatura de viajes relativa a las Islas Canarias hay algunas contribuciones que, a fuerza de ser utilizadas una y otra vez como recursos para acercarnos fielmente al pasado - como testimonios directos que fueron de una realidad vital hoy anhelada-, terminan por resultarnos cotidianas, habituales, incluso diríamos que hasta familiares. Nombres como los de Elizabeth Murray, Sabino Berthelot, René Verneau, André Pierre Ledru, Hermann Christ o el propio Alfred Samler Brown, por citar algunos entre tantos, traen a nuestra memoria retazos de este o de aquel episodio de la historia y del paisaje insular que fueron rescatados con primor por aquellos trotamundos durante su estancia isleña en el Ochocientos, y que lo hicieron a través de la pluma, pero también acompañada a veces del pincel, de la plumilla o de la cámara fotográfica.

No obstante, al focalizar sobre estos autores nuestra atención revertiendo ese paradigma y convirtiendo al visitante en objeto de introspección, cuando se trata de indagar las circunstancias particulares de esos mismos protagonistas tratando de saber algo más sobre sus vidas, sus intereses, la razón de su viaje a las Islas, no son pocas las ocasiones en que nos encontramos con una densa niebla de desconocimiento que cercena nuestros desvelos e inquietudes. En la mayor parte de las ocasiones se trata de biografías resueltas a base de trazos sencillos, con certeras indicaciones de sus profesiones, rangos o cargos, con las inapelables fechas de nacimiento y defunción, así como la identidad de sus maridos y esposas..., si bien existen casos en que ni siquiera se ha logrado avanzar mucho más allá del esclarecimiento del propio nombre y apellidos del visitante. En este sentido creemos que, si existe un viajero o viajera que ejemplarice esta peculiar situación de casi total anonimato, debemos acudir de forma insoslayable a la enigmática figura de Olivia M. Stone, cuya obra Tenerife and Its Six Satellites constituye uno de los referentes - posiblemente el más importante- dentro de la literatura extranjera sobre Canarias.

El libro de Stone ocupa meritoriamente una posición de privilegio en la bibliografía foránea sobre el Archipiélago ${ }^{1}$, y esta relevancia se fundamenta no solo

${ }^{1}$ García Pérez 1988: 175-184. Entre las primeras citas bibliográficas en las Islas que hacen alusión a Olivia Stone figura la de Elías Serra Ràfols, en su artículo de 1952 sobre los castillos betancurianos de Fuerteventura. Desde entonces la divulgación del texto de Stone y los estudios sobre él han conocido un notable desarrollo. Entre las aportaciones de los ochenta y los noventa están las de Morales Lezcano 1986; García Pérez 1988: 175-184; Lorente 1988; Díaz Almeida, Martel González, Naranjo Cigala y Murcia Suárez 1993; Vega de la Rosa 1994; González Cruz 1995 y 2017; González Lemus 1995, 1998. Entre las más recientes están las de Castillo 2000, 2002, 2008, 2010, y 2017; Hormiga Santana 2004; González Cruz 2011; Casañas Afonso 2013; García Pulido 2015; 
en el hecho de tratarse de una de las primeras obras en reflejar la descripción y visita puntual a todas y cada una de las siete islas, así como a la inmensa mayoría de sus poblaciones principales -lo que ya en sí constituye un auténtico hito y digno de ser alabado-, sino también en la variedad de los registros recopilados por la incansable viajera, con referencias que van desde el ámbito etnográfico, gastronómico o climatológico hasta datos históricos, sociodemográficos o sencillamente anecdóticos, todo en ese lenguaje característico y crítico asociado a la mirada victoriana del momento ${ }^{2}$.

Las primeras indicaciones acerca del peculiar anonimato que rodeaba la figura de Olivia M. Stone nos han llegado gracias al excepcional y precursor trabajo de investigación sobre los viajeros británicos efectuado por el catedrático José Luis García Pérez, quien ya nos avanzaba las causas de este desconocimiento: «Desgraciadamente hasta hoy ha sido imposible conocer algo de su biografía ya que al tomar el apellido de su marido todo rastro de su quehacer anterior ha quedado oculto» (1988: 175). Siguiendo esas directrices, parecía obvio que las únicas pautas conocidas acerca de esta viajera, cuya identidad familiar quedaba oculta debido a las normas de uso del apellido de su esposo, vinieran plasmadas en la portada y prolegómenos de la propia edición de Tenerife and Its Six satellites, editada en dos volúmenes en Londres en 1887 por Marcus Ward \& Co. Limited ${ }^{3}$, con una reedición corregida y aumentada dos años más tarde, en 1889, en un solo tomo. Estas páginas iniciales, así como la lectura de algunos pasajes del libro y las puntuales referencias en prensa que suscitó la aparición de esta importantísima obra ${ }^{4}$, constituían el único material sobre el cual fundamentar un proceso de búsqueda que nos ayudase a adentrarnos en la vida de Olivia M. Stone.

Del frontispicio anteriormente citado se rescata la identidad y la presencia cercana del marido de nuestra protagonista, de nombre John Harris Stone, abogado en ejercicio, MA (Master of Arts), FLS (Fellow of the Linnean Society) y FCS (Fellow of the Chemical Society), y de la dedicatoria misma de la obra surgen los nombres de los que, a primera vista, parecen ser los tres hijos de la pareja: Hartrick, Dudley y

y Beltrán Yanes 2017. Como testimonio fehaciente de la actualidad e importancia de esta viajera, durante el mes de febrero de 2017, en el Centro de Historia y Cultura Militar de Canarias, ubicado en el Fuerte de Almeyda, en Santa Cruz de Tenerife, se expuso la muestra "Olivia Stone y la naturaleza de las palabras», organizada por la Cátedra Cultural «Alexander von Humboldt» y el Vicerrectorado de Relaciones Universidad y Sociedad, de la Universidad de La Laguna, y comisariada por el profesor Francisco Javier Castillo.

${ }^{2}$ Véase la introducción de Jonathan Allen Hernández en la edición de la traducción española de la obra de Olivia Stone, donde se hace estudio pormenorizado de las pautas y características que definen la narración de esta singular viajera victoriana. STONE 1995: v-XXXII.

${ }_{3}$ Ubicada en Oriel House, en Farringdon Street, E.C., y con filiales en Belfast y Nueva York, como se especifica en la referida portada.

${ }^{4}$ En la introducción de su obra, la propia Olivia Stone (1995: I, 11) cita expresamente que «hemos escrito varios artículos relacionados directa o indirectamente con las Islas Canarias en muchos periódicos y revistas». García Pérez (1988: 175 y 180) cita, a su vez, varios textos surgidos a propósito de la edición de la obra, como el incluido en la sección «Literature» de The Atheneum (Londres, 24 de marzo de 1888) o la crítica referida a la pésima transcripción de los topónimos en la primera edición, firmada por George F. Hooper en The Academy en 1888. 


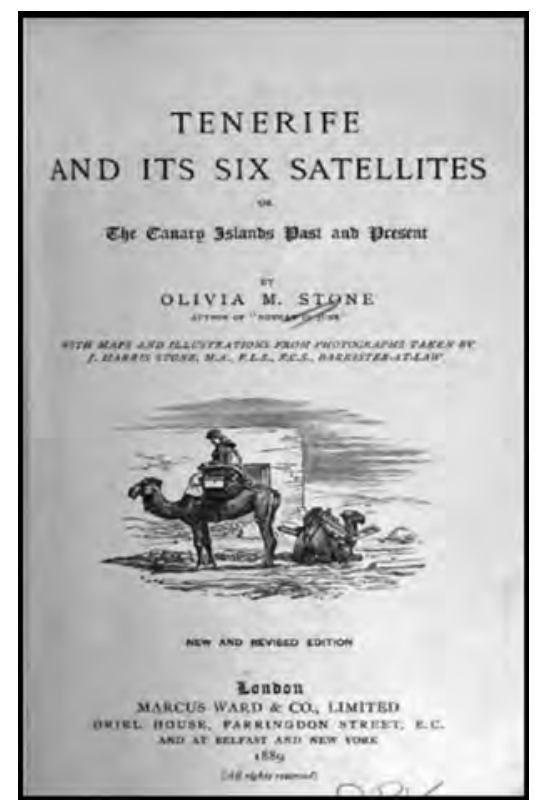

Portada de Tenerife and Its Six Satellites.

Erik $^{5}$. De la lectura atenta de la obra sobre las Islas pueden entresacarse referencias como la autoría por Olivia M. Stone, poco tiempo antes, en 1882, de otra narración de viajes, bajo el título Norway in June ${ }^{6}$; de la llegada a Tenerife a bordo del vapor francés Parana, de la Chargeur Reunis; o de la causa del retraso en la publicación de su trabajo, con una demora de casi tres ańos después de su estancia en las Islas. La viajera anota puntualmente en la introducción de Tenerife y sus seis satélites que todo fue "debido a una grave enfermedad que duró 18 meses, causada por el exceso de trabajo y mi preocupación por publicar estos volúmenes». ${ }^{7}$.

5 Olivia Stone menciona en el transcurso de su relato que llevaba con ella la fotografía de sus hijos -lo que demuestra que debieron de quedarse en Inglaterra al cuidado de familiares y personas de confianza-, que mostraba a todas aquellas mujeres isleñas de las que apunta que siempre tenían un aguzado interés por el tema de los niños y la infancia: «En todos los lugares donde nos quedábamos en las islas descubrí que las mujeres se interesaban enormemente por todo aquello relacionado con los niños y el placer llegaba a su punto más alto y llovían miles de preguntas cuando sacaba las fotografías de los míos» (Stone 1995: 121-122).

${ }^{6}$ Stone 1995: I, 10. En varios pasajes reitera haber viajado a las islas del Canal de la Mancha -particularmente, Jersey y Sark-, a Noruega y a Islandia (vol. I, 29, 138, 155, 224, 277 y 308) e incluso deja entrever haber visitado Suiza, Nueva Zelanda, Estados Unidos e incluso Canadá (vol. I, 132, 293 y 471; II, 8 y 68).

7 Stone 1995: I, 10. A modo de curiosidad cita que en La Laguna se quedó en una posada «enfrente del fielato», no lejos del centro de la ciudad. 


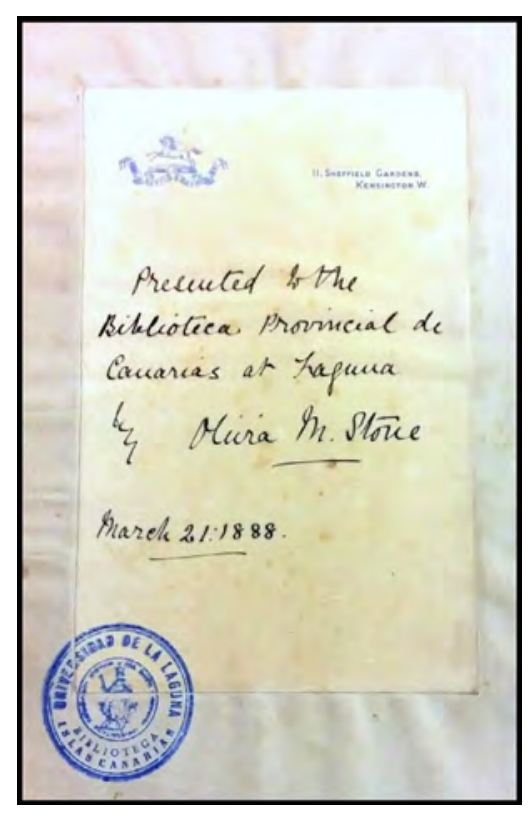

Dedicatoria de Olivia Stone a la Biblioteca Provincial de Canarias.

A lo largo del texto Olivia Stone efectúa constantes insinuaciones y guiños a la tierra irlandesa -como avanzando de facto su naturaleza y origen de dicha región $-{ }^{8}$, y nos presenta al círculo de colaboradores y amistades que estableció en el tiempo que permaneció en las Islas y en los años en que estuvo preparando la edición, con personas que podríamos subdividir en varias categorías: por un lado, el entorno consular, como los vicecónsules John Howard Edwards (Tenerife), James Miller (Gran Canaria) o John Thomas Topham (Lanzarote); por otro, la colonia británica o anglófila afincada en las Islas, como John y Hugh Hamilton, Mr. Reid, John Swanston o George John Graham-Toler; a continuación, un elenco de personalidades europeas o extranjeras residentes en tierra canaria como Hermann Wildpret, George B. Nixon o Arthur Henry Bechervaise; y, obviamente, los miembros de la burguesía

8 Stone 1995. Hay ejemplos en vol. I., 64, citando la prenda irlandesa cothamore en comparación a la manta esperancera; p. 83, a propósito de la analogía isleńa de cabalgar con albardas en el lado derecho de las bestias, "como si montasen un carruaje irlandés»; pp. 215 o 253, donde el musgo y el liquen herreńos le recuerdan a la «isla Esmeralda»; o en la pp. 274, 342, 471 y 491, donde el paisaje isleño le recuerda la Calzada del Gigante, en Antrim, Irlanda del Norte. En la p. 455 se permite hacer una loa del pueblo irlandés, «uno de los más virtuosos de la tierra, por no decir el más». En el vol. II hay citas a Irlanda en las pp. 40-41, 43, 46, 59, 146, 166, 249 (recordando el río Dargle, al sur de Dublín), 301, 389 y 404. Llega a afirmar que semillas del tagasaste isleño las había plantado «en Inglaterra e Irlanda», demostrando su vinculación con ambas naciones (vol. I, 351). 
y nobleza isleñas que no dejaron de atender al matrimonio Stone durante su estancia: Lorenzo Machado Benítez de Lugo, Alberto Cólogan Cólogan, Luis Renshaw de Orea, Juan de la Guardia o Gregorio Chil y Naranjo (García Pérez 1988: 177, 182 y 183). Como refuerzo de este vínculo afectivo y de agradecimiento con el Archipiélago no podemos soslayar las entrańables palabras que dirige a los canarios, que no nos resistimos a copiar en este punto:

Quisiera decirles a todos mis amigos insulares que puedan leer este libro que todo lo escrito, ya sea sobre sus virtudes o defectos, siempre lo he hecho inspirada por mi aprecio por los habitantes y mi amor por su feliz patria, las Islas Afortunadas (Stone 1995: I, 12).

Siempre recordaremos las islas como las vimos nosotros -verdaderamente unas Islas Afortunadas-, lo más cercano a cualquier Paraíso Terrenal que un Morris pudiera cantar o un Tadema pintar (Stone 1995: II, 465).

Atendiendo a estas escuetas referencias parecía obvio que, a fin de conocer alguna referencia más acerca de la vida y dilucidar la identidad de Olivia M. Stone, debíamos bucear en la biografía de su esposo, John Frederick Matthias Harris Stone, o sencillamente J. Harris Stone, como le gustaba que lo llamasen'. Los anales británicos son fecundos sobre su persona y nos detallan que había nacido en la localidad de Bath (Somerset), en 1853, siendo el séptimo de un total de siete hijos en la familia conformada por el procurador y secretario del Ayuntamiento de aquella ciudad, John Stone (1818-1899), auténtica personalidad en el ámbito local ${ }^{10}$. Activo colaborador en prensa y reconocido abogado -tras haber estudiado en la Dame's School y en el Clifton College, ambos en Bath ${ }^{11}$, y con titulación académica en Ciencias Naturales obtenida en la siempre prestigiosa Universidad de Cambridge-, John Harris tomó parte activa desde su juventud en la vida social del momento y prueba notable de ello es que no solo en 1885 fundó, movido por sus querencias hacia el

9 Cuando ya ultimábamos la redacción del presente artículo y a través del testimonio del profesor Francisco Javier Castillo hemos sabido que el licenciado Iru Jesús Rodríguez Navarro ha presentado un trabajo de fin de grado - aún inédito-, defendido en julio de 2014, sobre la figura de Olivia Stone, donde recoge igualmente múltiples referencias acerca de su biografía y antecedentes familiares.

${ }_{10}$ Autor de un diario desde noviembre de 1838 a enero de 1899 , continuado en sus últimos años por su hijo John Harris, hoy depositado en la Bath Municipal Library (British Diaries: an annotated bibliography of British diaries written between 1442-1942. Ed. William Matthews; University California Press, 1984). La prensa de Bath confirma que una sociedad en la que él participaba como procurador desde 1855, conocida como «Stone King», aún existe hoy en día. Los nombres de los hermanos de John Harris eran Louisa, Deborah, Mary, Henry, William y Ethel. Para mayor coincidencia, Olivia Stone, en su referida obra sobre las Islas, utiliza esta localidad como ejemplo de su discurso narrativo, evidenciado así la asiduidad y cercanía con ese enclave: «Usando solo el mapa y la regla -como el ferrocarril entre Londres y Bath-» (Stone 1995: 70-71) y compara la catedral de Santa Ana, en Las Palmas de Gran Canaria, con la abadía de dicha localidad británica (vol. II, 12).

${ }^{11}$ Curiosamente, este Clifton College tiene una biblioteca de ciencias bautizada como Stone Library en honor de los hermanos Stone (Ellis 2006). En su estancia en Tenerife coincidieron los Stone con un «viejo amigo de la British Association», la célebre academia británica de las ciencias (vol. I, 323), a quien lamentablemente no identifican en el texto. 


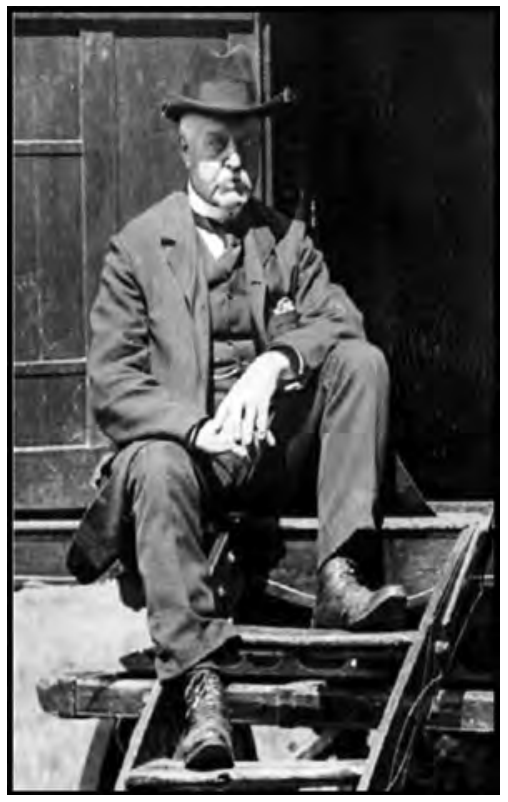

John Harris Stone.

Cortesía de The Caravan Club.

mundo de la fotografía, la sociedad The Camera Club, con sede en Bedford Street, en el Strand londinense, llegando a ser editor de una revista fotográfica amateur ${ }^{12}$, sino que pocos años después impulsó la creación del Caravan Club, sociedad de la que sería secretario honorario desde 1907 a 1935 y vicepresidente desde 1935 a 1939.

De su biografía destaca sobremanera la gran cantidad de publicaciones que realizó, con títulos tan sugerentes como Physiology of every-day Life: a lecture (Londres y Belfast, 1881); Nihilist Napoleon. A tale (1888, junto a Percy Carter); The civic reader (1893, junto a B. Johnson); Local government act. A practical ready reference guide to the election of parish and rural district councillors (1894, junto a Joseph Gerald Pease); Achill Island, Dugurt and Dooagh (1906) ${ }^{13}$; Connemara and the neighboring spots of beauty and interest (Londres, 1906); England's Riviera, a topographical and archaelogical description of Land's End, Cornwall and adjacent spots of beauty and interest (Londres, 1912); y Caravanning and camping out: experiences and adventures

${ }^{12}$ Debe constar aquí nuestro agradecimiento a Kevin Birch, presidente, y Michael Colman, archivero, del The Camera Club, Londres, por brindarnos fuentes de información acerca de John Harris Stone.

${ }^{13}$ En la introducción de esta obra dejaba patente que seguía influenciado por el espíritu que motivó el conocido viaje a las Islas Canarias: «En estas páginas únicamente me he esforzado, puede que de forma inadecuada, en describir a través de la pluma y de la lente el oeste de Irlanda tal y como yo lo contemplé». 
in a living-van and in the open air, with hints and facts (Londres, 1913). Asimismo llama la atención su sentido patriótico, cuando, con motivo del estallido de la Primera Guerra Mundial, quiso contribuir a la causa de la defensa nacional y se alistó en el Civil Service como secretario interino en el Departamento de Control de Carburantes. Nuestro biografiado fallecería, a la avanzada edad de 86 años, en $1939^{14}$.

De forma complementaria a estos datos biográficos del esposo de Olivia M. Stone, gracias al inestimable recurso de los investigadores genealógicos británicos ${ }^{15}$, hemos tenido acceso al censo efectuado en el territorio británico en 1881, fuente de valor inestimable que nos abrirá definitivamente el camino para conocer su apellido de soltera y, con ello, su origen familiar. En el empadronamiento aparece fielmente reflejada la pareja objeto de nuestras inquietudes viviendo en el n. ${ }^{\circ} 11$ de Sheffield Gardens, en Kensington, en la capital de Londres ${ }^{16}$, bajo la siguiente descripción:

- John Harris Stone, natural de Bath, de 27 años.

- Olivia Mary [Hartrick] Stone, natural de Irlanda, de 25 años.

- Mary Macaulay Hartrick, natural de Irlanda, de 71 años. Suegra del titular.

- Dos niños: John Hartrick Stone, de 1 año; y un recién nacido, del que no consta siquiera su nombre.

- Cocinera, Beatrice Barrow, inglesa, de 17 años.

- Enfermera doméstica, Annie McG. Simpson, irlandesa, de 26 años.

La mención del apellido Hartrick nos hizo avanzar en ese sentido para conocer la ascendencia familiar de Olivia Stone, encabezada hasta donde hemos podido saber por John Hartrick, labrador establecido en la localidad irlandesa de New Ross, en Wexford, quien tuvo, al menos, un hijo, el reverendo Edward John Hartrick (1812-1893), M.A. y T.C.D. Este se desposó en 1842 con la citada anteriormente en el censo Mary Macaulay Dobbs (1819-1892), hija a su vez del reverendo Richard Stewart Dobbs. Esta pareja procrearía hasta cinco hijos:

- Richard Stewart Hartrick (1845-j...?), C.E., que se desposaría en 1872 con Mary B. Culbertson, hija de Albert Culbertson, de Monongahala City,

${ }_{14}$ Necrológica en Proceedings of the Linnean Society of London, vol. 151, n. ${ }^{\circ}$ 4, mayo 1941. Datos biográficos obtenidos en parte en el censo británico de 1881 y en otras fuentes que nos ha brindado el Caravan Club (National Motor Museum, en Brokenhurst, Hampstead), a quienes agradecemos fervientemente la información. Al fallecer John Harris Stone era poseedor de un interesante legado documental, hoy guardado en la East Grinstead House (Ellis 2006).

${ }^{15}$ Debemos la obtención y registro de estas referencias genealógico-históricas a los investigadores Mike Hartrick y Paul Robinson.

${ }^{16}$ Esta misma dirección (11, Sheffield Gardens, Kensington) aparece claramente visible, como membrete impreso, en la hoja de dedicatoria manuscrita que, pegada al lomo de uno de los tomos de la obra de Olivia Stone, figura en el ejemplar donado por la autora a la entonces Biblioteca Provincial del Instituto de Canarias, en la actualidad en los fondos de la Biblioteca Universitaria de La Laguna. 


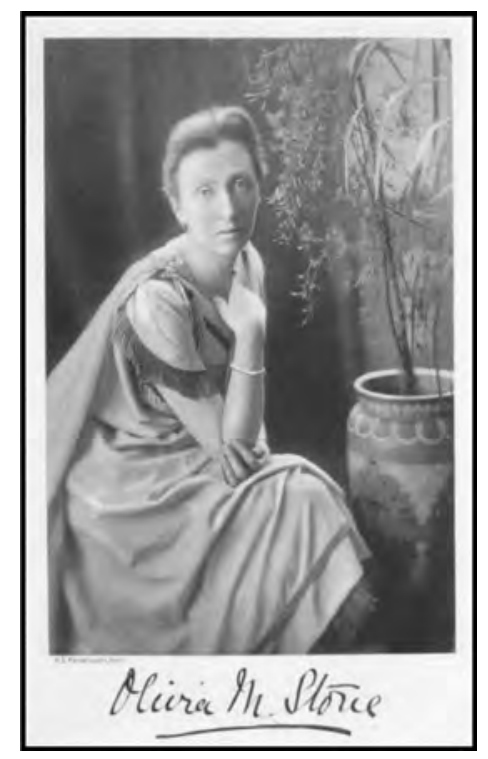

Biblioteca Universitaria de La Laguna.

Pensilvania, que fallecería en Pittsburg en 1911, padres ambos de James Albert Hartrick.

- Edward Macaulay Hartrick (1847-1915), C.E., que casaría en 1870 con Mary McClelland, y falleció en Dickinson, Galveztown, Texas, siendo enterrado en el Holy Trinity Episcopal Church Cemetery.

- Olivia Mary Hartrick (1855-¿...?), nuestra Olivia M. Stone.

- Francis Henry Hartrick (1851-56).

- John Edward Hartrick (1843-50), fallecidos ambos a temprana edad.

Los recursos genealógicos nos han permitido conocer múltiples aspectos de la vida personal de estos personajes, entre los que destaca que el casamiento de John Harris y Olivia Stone se produjo en 1878; que posiblemente la localidad de nacimiento de Olivia sea Wexford, en Irlanda -acaso la misma población de New Ross, donde estaba establecido su abuelo John Hartrick- ${ }^{17}$; y que los hijos de la pareja fueron:

- El teniente John Hartrick Stone, del Royal Field of Artillery, que se desposó en la iglesia de St. Mary's, en Stamford Brook-Hammersmith, Londres- el

17 En este punto no podemos soslayar hacer referencia a otra coincidencia enriquecedora que figura en la obra de Olivia Stone (II, 333-334). Al relatar el triste episodio del asesinato de George Glas en las costas de Irlanda nos apunta que estos hechos tuvieron lugar en Duncannon, muy cerca de Wexford, y continúa diciendo que ha «llevado a cabo toda investigación posible tanto en el condado de Wexford como en el de Waterford», demostrando así su familiaridad con este territorio. 
3 de noviembre de 1906 con Florence Harriet Glyn Wellbeloved, nacida en 1886, hija de Richard Wellbeloved.

- Eric Edward Stone, nacido en Larne el 20 de agosto de 1882 y muerto el 1 de octubre de 1918, casado con Mabel Stone, que fallecería a su vez el 14 de julio de 1932. Esta pareja tuvo un recién nacido, muerto prematuramente el 26 de diciembre de 1912 en Dickinson, Texas.

- Dudley Stone, del que nada sabemos.

Ahondando en la documentación incluso hemos podido saber que John Harris Stone se desposó por segunda vez el 29 de diciembre de 1900 en la iglesia de St. Marylebone, en Londres, con Lillie Tully -hija del ingeniero Thomas Dominic Tully-, prueba evidente de que Olivia Stone debió haber fallecido en el transcurso del último decenio del siglo $\mathrm{XIX}^{18}$. Si a este cúmulo de indicaciones ańadimos los datos que sobre la vida del matrimonio Stone tras su regreso a Inglaterra después de su periplo isleño (González Cruz 2011:81) ha obtenido la profesora María Isabel González Cruz, tenemos que «de Olivia M. Stone sabemos que vivía junto a su marido, John Harris Stone, en una casa en Dover (Kent), a la que habían puesto el nombre de Fuerteventura, y que tenían decorada con abundantes recuerdos de Canarias». Así pues, contamos con referencias para rastrear en un futuro próximo la fecha de defunción de nuestra ańorada viajera ${ }^{19}$.

Como conclusión, no deberíamos cejar en adentrarnos más y más en el conocimiento de los detalles de esa interesantísima parcela de nuestra idiosincrasia constituida por la recopilación de las narraciones de todos aquellos viajeros y viajeras que hicieron estadía en el Archipiélago y nos legaron descripciones, apuntes o referencias que constituyen hoy parte de nuestro acervo patrimonial. En el amplio imaginario dibujado por la historiografía canaria existen multitud de temáticas por desarrollar, de enigmas o confusiones por esclarecer, de espacios en blanco esperando ser rellenados con el paso del tiempo, conforme vayan surgiendo nuevas fuentes documentales y detalles rescatados de algún papel desconocido hasta la fecha, de una memoria revisada, de una mirada inquieta. La identidad de Olivia Mary Hartrick Stone era una deuda pendiente con una viajera que nos ha legado, posiblemente, una de las mejores obras de literatura de viajes existentes sobre las Islas. Saldar ese débito de memoria ojalá sirva en parte de sincero reconocimiento al recuerdo y la figura de un espíritu privilegiado en una época de cambios, de contrastes, de crecimiento.

Recibido: febrero de 2019; ACEPTADO: junio de 2019.

${ }_{18}$ No deja de resultar tremendamente curioso que en 7 de noviembre de 1895 llegase otro individuo apellidado Stone a Santa Cruz de Tenerife a bordo del Wazzan, procedente de Londres y Funchal (La Opinión, Santa Cruz de Tenerife, 9 de noviembre de 1895).

${ }_{19}$ Esa residencia en Dover parece confirmarse con otro pequeño trabajo publicado en 1910 por John Harris Stone sobre St. Margaret's Bay, a unas ocho millas al este de dicho Dover, enclave al que él llamaba particularmente como la «Picadilly de los mares». 


\section{BIBLIOGRAFÍA}

Beltrán Yanes, Esther (2017): «El Teide en la obra de Olivia Stone: el paisaje como recurso turístico", Cuadernos de Turismo 39: 67-89.

Casañas Afonso, Roberto (2013): «Olivia Stone, la mirada de una turista en el Lanzarote de finales del siglo XIX», Jameos 19: 28-31.

Castillo, Francisco Javier (2000): «La ciudad de las campanas, los serenos y la lluvia. La Laguna a finales de 1883", El Día/La Prensa (Santa Cruz de Tenerife), 19 de febrero.

CAstillo, Francisco Javier (2002): «Literatura de viajes y realidad insular: cuestiones de idealidad y de procedimiento", Nerter 3-4: 96-100.

Castillo, Francisco Javier (2008): «Humboldt en la literatura inglesa de viajes», en Belén Castro Morales (coord.), Actas del simposio Alexander von Humboldt entre volcanes (La Laguna, 3-5 mayo 2007), Servicio de Publicaciones de la Universidad de La Laguna, 219-233.

Castillo, Francisco Javier (2010): «Las hablas insulares en la literatura inglesa de viajes», en Dolores García Padrón y María del Carmen Fumero Pérez (coords.), Tendencias en lingüistica general y aplicada, Frankfurt am Main: Peter Lang, 53-62.

Castillo, Francisco Javier (2017): «Sobre la literatura de viajes en la etapa victoriana. El Atlántico cercano en Olivia Stone», Revista de Filología (Universidad de La Laguna), 35, pp. 73-105.

Díaz Almeida, Francisco Luciano, F. Martel González, A. Naranjo Cigala y M. Murcia SuáREZ (1993): «El viaje como rito: Olivia Stone en Lanzarote. Un ensayo de geografía de la percepción», Boletín Millares Carlo 12: 105-117.

Edwardes, Charles (1888): Rides and studies in the Canary Islands, London: T. Fisher Unwin.

Edwardes, Charles (1998): Excursiones y estudios en las Islas Canarias, traducción y notas de Pedro Arbona, Las Palmas de Gran Canaria: Ediciones del Cabildo Insular de Gran Canaria.

Ellis, C. (2006): The history of the Caravan Club, East Grinstead.

García PéreZ, José Luis (1988): Viajeros ingleses en las Islas Canarias durante el siglo XIX, Santa Cruz de Tenerife: Caja General de Ahorros de Canarias.

García Pulido, Daniel (2015): «Olivia M. Stone: aproximación a una biografía desconocida», $E l$ Día / La Prensa (Santa Cruz de Tenerife), 15 de febrero, 1-3.

GonzÁlez Cruz, María Isabel (1995): La convivencia anglocanaria. Estudio sociocultural y lingüístico (1880-1914), Las Palmas de Gran Canaria: Ediciones del Cabildo Insular de Gran Canaria.

GonzÁlez Cruz, María Isabel (2011): «Hispanismos y canarismos en los textos de dos viajeras inglesas decimonónicas», Revista de Filología (Universidad de La Laguna), 29: 81-99.

González Cruz, María Isabel (2017): «Olivia M. Stone y la conservación del patrimonio prehispánico", en Flavia Aragón Rosano y José Antonio López Sánchez (eds.), Historias de viajes: una perspectiva plural, Peter Lang, 103-117.

González Lemus, Nicolás (1995): Las islas de la ilusión (Británicos en Tenerife. 1850-1900), Las Palmas de Gran Canaria: Ediciones del Cabildo Insular de Gran Canaria.

GonzÁlez Lemus, Nicolás (1998): Viajeros victorianos en Canarias. Imágenes de la sociedad isleña en la prosa de viajes, Las Palmas de Gran Canaria: Ediciones del Cabildo Insular de Gran Canaria.

Hormiga Santana, Marcos (2004): «La visión anglosajona sobre Fuerteventura y Lanzarote», en XI Jornadas de Estudios sobre Fuerteventura y Lanzarote, vol. II, Puerto del Rosario: Cabildo Insular de Fuerteventura y Cabildo Insular de Lanzarote. 369-397. 
Lorente, María José (1988): «El viaje de Olivia Stone, noviembre 1883, febrero 1884», en Víctor Morales Lezcano (coord. y pról.), II Aula Canarias y el Noroeste de África, [Las Palmas de Gran Canaria]: Cabildo Insular de Gran Canaria, 287-309.

Morales Lezcano, Víctor (1986): «Canarias, Madeira y Azores en la literatura de viajes inglesa del siglo XIX», Anuario de Estudios Atlánticos, 32: 525-529.

Rodríguez Navarro, Iru Jesús (2014): The land beyond the city. On $19^{\text {th }}$ century English travel literature on the Canaries, trabajo de fin de grado dirigido por el profesor Francisco Javier Castillo, Facultad de Humanidades-Sección de Filología, Universidad de La Laguna, inédito.

SERra RÀfols, Elías (1952): «Los castillos betancurianos de Fuerteventura», Revista de Historia (Universidad de La Laguna), tomo XviII, n. ${ }^{\circ}$ 100, 509-527.

Stone, Olivia M. (1882): Norway in June, London: Marcus Ward.

StOne, Olivia M. (1887): Tenerife and Its Six Satellites, 2 vols., London: Marcus Ward \& Co., Limited.

Stone, Olivia M. (1889): Tenerife and Its Six Satellites, new and revised edition, London: Marcus Ward \& Co., Limited.

Stone, Olivia M. (1995): Tenerife y sus seis satélites, 2 vols., introducción y revisión de Jonathan Allen Hernández; traducción y notas de Juan S. Amador Bedford, Las Palmas de Gran Canaria: Cabildo Insular.

Vega de la Rosa, Carmelo (1994): «Viaje fotográfico a un archipiélago inédito: las imágenes de J. Harris Stone para el libro Tenerife and Its Six Satellites (1887)", en Francisco Morales Padrón (coord. y pról.), XI Coloquio de Historia Canario-Americana, vol. 2, Las Palmas de Gran Canaria: Cabildo Insular de Gran Canaria, 393-408. 